\title{
El diseño y desarrollo de animaciones como estrategia que ayuda a mediar la comprensión del equilibrio químico en la escuela
}

\author{
The design and development of animations as a strategy that helps mediate the \\ understanding of chemical equilibrium in school
}

Boris Fernando Candela Rodríguez

boris.candela@correounivalle.edu.co

Universidad del Valle (Colombia)

\section{Resumen}

El propósito de este estudio es investigar si el diseño y desarrollo de animaciones mediadas por la técnica del Stop Motion, ayudan a los estudiantes en la construcción de una comprensión del equilibrio químico a nivel molecular. La metodología fue cualitativa por estudio de casos, y los datos se recogieron por medio de fuentes documentales como: videos de clases, observación no participante, trabajos de los estudiantes, y entrevistas. El análisis de los datos generó las siguientes categorías: (1) El diseño de animaciones en conjunción con una orientación de enseñanza sociocultural y fenomenológica, factor clave en la diferenciación e integración de los niveles de representación de la química; (2) El par evaluador de las animaciones elemento clave en la construcción de la compresión del equilibrio químico; (3) Las animaciones herramienta cognoscitiva que apoya la visualización, interpretación y razonamiento de los procesos químicos; y (4) El diseño de las animaciones factor importante en el desarrollo de las habilidades de pensamiento que demanda la sociedad del conocimiento. Estos resultados permitieron concluir que el diseño y desarrollo de animaciones a través de la técnica del Stop Motion dentro de un contexto de enseñanza de perspectiva sociocultural, ayudaron a mediar la comprensión del equilibrio químico.

Palabras clave: animaciones, visualización, Stop motion; educación en química; equilibrio químico.

\begin{abstract}
The purpose of this study is to investigate whether the design and development of animations mediated by the Stop Motion technique, help students to build an understanding of chemical balance at the molecular level. The methodology was qualitative by case study, and the data was collected through documentary sources such as: class videos, non-participant observation, student work, and interviews. The analysis of the data generated the following categories: (1) The design of animations in conjunction with a sociocultural and phenomenological teaching orientation, a key factor in the differentiation and integration of the levels of representation of chemistry; (2) The evaluating pair of animations is a key element in the construction of chemical equilibrium compression; (3) Animations cognitive tool that supports the visualization, interpretation and reasoning of chemical processes; and (4) The design of animations is an important factor in the development of thinking skills demanded by the knowledge society. These results allowed us to conclude that the design and development of animations through the Stop Motion technique within a sociocultural perspective teaching context, helped to mediate the understanding of chemical balance.
\end{abstract}

Keywords: animations, visualization, Stop motion; chemistry education; chemical balance. 


\section{INTRODUCCIÓN.}

En el campo de la educación en química se ha llegado al consenso que muchos de los estudiantes graduados de la escuela secundaria aún sostienen fuertes concepciones alternativas de los fenómenos químicos, a pesar de haber sido intervenidos desde una enseñanza de perspectiva constructivista (Chang y Quintana, 2006; Candela y Viafara, 2014). De hecho, los resultados de varias pruebas estandarizadas aplicadas en diferentes países dejan ver que, de todas las áreas evaluadas la educación en química, es una de las disciplinas donde los aprendices obtienen los menores rendimientos.

Así mismo, se ha evidenciado que los estudiantes de la escuela secundaria típicamente no tienen las habilidades representacionales o discursivas que caracterizan las prácticas de investigación de los químicos (Kozma, 2000). Desde luego, dichas habilidades les permiten a estos profesionales representar de manera submicroscópica y simbólica, los diferentes cambios percibidos en la apariencia de las sustancias, con la intención de comunicar a sus pares la comprensión acerca de los procesos dinámicos que subyacen a tales transformaciones macroscópicas. Por esto, se considera que la formulación de una explicación sobre las transformaciones físicas o químicas que sufren las sustancias, implica la diferenciación e integración de los niveles de representación macroscópico, submicroscópico y simbólico que caracteriza el sistema semiótico de la química (Candela y Viafara, 2014).

Este sistema de representación junto con el discurso y las actividades de creación de significado de la comunidad científica, han dado lugar a avances significativos en la comprensión de constructos químicos, tales como: geometría molecular, transformaciones físicas y químicas, cinética y equilibrio químico, entre otros (Candelas, 2018). Por todo esto, dichas representaciones se encuentran entre los sistemas físicos históricamente construidos por los científicos, con miras apoyar la comprensión de las entidades y procesos que subyacen a los fenómenos químicos.

Por otro lado, la investigación en educación en química ha determinado que las restricciones que tienen los estudiantes en la diferenciación e integración de los tres niveles de representación, acentúa las concepciones alternativas con las que llegan al aprendizaje de los contenidos de esta disciplina. Por ejemplo, se ha evidenciado que la comprensión del fenómeno del equilibrio químico por parte de los estudiantes resulta ser muy compleja de alcanzar, dado que, ellos presentan serias dificultades en el establecimiento de conexiones profundas entre la realidad, el mundo molecular y el sistema de fórmulas y ecuaciones químicas (Chin y Wu, 2009).

Ahora bien, en los últimos años se ha inferido que las entidades y procesos que subyacen al fenómeno del equilibrio químico, pueden ser representados y formulados de manera apropiada a través de técnicas de visualización como las animaciones, a fin de asistir a los estudiantes en la construcción de una profunda comprensión del fenómeno (Chan y Black, 2005). Así pues, estas herramientas cognitivas se caracterizan por representar un fenómeno natural, social o artificial por medio de un conjunto de imágenes en constante movimiento (Hoban y Nielsen 2010). De donde se infiere, que esta clase de recurso multimedia puede hacer visible los contenidos químicos de niveles de abstracción altos, especialmente aquellos relacionados con la cinética molecular de las sustancias. 
Así mismo, Weiss, Knowlton y Morrison (2002) afirman que las animaciones dentro del proceso de enseñanza pueden cumplir las siguientes funciones: (1) funciones decorativas, hacen que la instrucción sea atractiva para los estudiantes; (2) funciones de atención, señalan puntos destacados de un contenido; (3) funciones de motivación, proporcionan retroalimentación a fin de reforzar las respuestas correctas; (4) funciones de representación, proporcionan una referencia concreta y un contexto visual para las ideas; y (5) funciones de aclaración, esclarecen las relaciones entre los elementos que configuran el fenómeno representado a través de medios visuales. También, ellos resaltan que el uso de este tipo de recurso curricular ha estado focalizado en las tres primeras funciones (enfoque de visualización), las cuales se han preocupado por visualizarles a los aprendices un fenómeno natural con la intención de que puedan comprenderlo.

Sin embargo, Hubscher-Younger y Narayanan, (2003) argumentan que mostrar una animación de un fenómeno químico a los estudiantes, no es suficiente para que puedan construir una comprensión profunda de lo representado. Desde luego, este tipo de recurso usado de esta forma, se caracteriza por presentar los procesos moleculares de manera muy rápida, situación que limita la identificación y establecimiento de las relaciones conceptuales que fundamenta el fenómeno en cuestión (Hoban y Nielsen 2010). De ahí que, los investigadores hayan comenzado a abordar métodos alternativos de usar esta herramienta digital con miras a asistir a los aprendices en la comprensión del fenómeno. Estas perspectivas se centran en hacer uso de las animaciones como herramientas constructivistas, que respaldan la visualización e interpretación de procesos abstractos por parte de los estudiantes y; dispositivos que ayudan a mediar la resolución de problemas apoyando los procesos de razonamiento de los aprendices (Vermaat, Kramers-Pals y Schank, 2003; Hoban y Nielsen 2010).

En este sentido, Chang, Quintana y Krajcik, (2010) afirman que las tareas donde se les pide a los estudiantes que diseñen y evalúen animaciones, apoyándose en la diferenciación e integración de los tres niveles de representación de la química (macroscópico, submicroscópico y simbólico), resultan ser efectivas en la compresión de los fenómenos químicos. Naturalmente, el diseño, desarrollo y evaluación de las animaciones fomenta una enseñanza centrada en los estudiantes y un aprendizaje activo. En esta clase de tareas cognitivas los aprendices emplean estrategias tales como la selección y organización de la información, y el establecimiento de conexiones entre la experiencia previa y el nuevo conocimiento (Chan y Black, 2005).

De hecho, cuando los estudiantes utilizan herramientas digitales generales o especificas a la tarea (ej., Stop Motion, Chemsense animatior, Chemation, Molecular Workbench, entre otros), con miras a construir animaciones sobre un fenómeno químico a nivel molecular, tienen que visualizar las entidades y los procesos químicos dinámicos que subyacen al fenómeno bajo consideración (Hoban y Nielsen 2010; Hoban, Loughran y Nielsen, 2011). Sin embargo, resulta conveniente destacar que el acceso y uso de los paquetes de software diseñados concretamente para representar contenidos de la química, en muchas ocasiones resulta limitado y complejo de alcanzar por estudiantes de niveles socioeconómicos bajos. De ahí que, Hoban, et al. (2011) argumentan que existen en la Internet software de naturaleza genérica y de uso libre, que se encuentran alineado con la técnica de animación del Stop Motion, cuya estructura lógica brinda la oportunidad a los estudiantes de diseñar y 
desarrollar animaciones de manera simple a fin de representar el fenómeno químico en consideración.

Desde luego, la técnica de animación del Stop Motion tiene las potencialidades de ayudar a los estudiantes a conectar fenómenos macroscópicos con representaciones submicroscópicas, a fin de explicar los fenómenos químicos en términos moleculares (Hoban y Nielsen 2010; Hoban, et al., 2011). Por tanto, el uso de esta técnica de visualización demanda de los aprendices un pensamiento de orden superior, que les permite establecer una conexión entre las observaciones producidas por las transformaciones químicas o físicas de las sustancias, y las inferencias que explican y predicen dichos cambios. Definitivamente, llegar a la construcción compartida del contenido químico implica que los estudiantes realicen procesos de planificación, diseño, desarrollo y evaluación de la animación en consideración (Chin y Wu, 2009; Hoban, et al., 2011). Finalmente, el propósito de este estudio es investigar si el diseño y desarrollo de animaciones mediadas por la técnica del Stop Motion, ayudan a los estudiantes en la construcción de una comprensión del equilibrio químico a nivel molecular. A partir de estos presupuestos se formuló el siguiente interrogante de investigación:

¿Cómo el diseño y desarrollo de una animación a través de la técnica de visualización del Stop Motion, ayuda a mediar la comprensión de las entidades y procesos que subyacen al fenómeno del equilibrio químico?

\section{MÉTODOS}

La metodología que direccionó este estudio es de perspectiva cualitativa por estudio de casos (Yin, 2003). Este paradigma de indagación permitió construir una teoría naturalística, acerca de cómo el diseño y desarrollo de animaciones a través de la técnica del Stop Motion junto con los razonamientos y acciones pedagógicas del profesor, asistieron a los estudiantes de undécimo grado en la construcción de la comprensión del equilibrio químico.

Conviene subrayar que, la implementación de la secuencia de actividades de aprendizaje se realizó del 1 de agosto al 13 de septiembre del 2018 (tres horas semanales de clase). Durante este período los datos fueron recogidos por fuentes documentales, tales como: videos de clases, artefactos diseñados por los estudiantes y entrevista semiestructurada a los aprendices. Así, los videos fueron grabados por dos cámaras, una fija y otra rotando por el aula que fue manejada por el asistente de investigación, con el fin de documentar los eventos críticos estrechamente vinculados con la problemática estudiada (10 videos de hora y media de duración). Los artefactos digitales producidos por los aprendices en el marco de la experimentación, fueron obtenidos como resultado de la necesidad de dar solución a la serie de tareas problemas, las cuales configuraron la secuencia de actividades de aprendizaje sobre el equilibrio químico. La recolección de estas composiciones digitales tuvieron como fin, lograr evidenciar, la evolución progresiva de los modelos mentales de los estudiantes acerca del fenómeno químico estudiando.

Por otro lado, la entrevista semiestructurada estuvo configurada por varías preguntas abiertas que se encontraban estrechamente relacionadas con el eje problemático, y cuyo 
propósito fue el de estimular a los estudiantes a pensar en voz alta acerca de los principales elementos que estructuraron el proceso de enseñanza y aprendizaje del fenómeno del equilibrio químico en el marco de la experimentación y la modelación. Dichos interrogantes fueron reformulados en dos ocasiones, como consecuencia a las sugerencias realizadas por dos investigadores, quienes son reconocidos por la comunidad universitaria como expertos en el campo de la Educación en ciencias. Una vez finalizada la implementación de la lección, la entrevista fue realizada por el investigador a varios estudiantes seleccionados de manera aleatoria. Adicionalmente, él les generó otros interrogantes in situ, con la intención de recoger información pertinente para la comprensión del caso estudiado.

La selección del caso estuvo orientada por los criterios de accesibilidad y disponibilidad de los sujetos en el desarrollo de las tareas de investigación. En este sentido, se buscó un profesor de química experimentado de grado once, y conocedor de las potencialidades que ofrece la técnica del Stop Motion para representar a nivel molecular los fenómenos químicos, además, que tuviera un apropiado Conocimiento Didáctico del Contenido de la química con el fin de andamiar el aprendizaje de los estudiantes. Así pues, estos criterios permitieron seleccionar a un profesor graduado en educación en química y con estudios de maestría en enseñanza de las ciencias, quien lleva 28 años planeado y enseñando los contenidos del currículo de esta disciplina. Otro elemento que configura el caso estudiado hace referencia a los 24 estudiantes, con una edad promedio de 16 años (18 niñas y 6 niños), y quienes cursaban el grado once en una escuela pública de la zona urbana del municipio de Santiago de Cali (Colombia). Una característica que medió la elección de los estudiantes, fue que ellos previamente habían desarrollado en su curso de informática las competencias en el manejo del Stop Motión o técnica de visualización. Conviene subrayar que, por razones de ética, en esta investigación los nombres de los sujetos estudiados e institución fueron modificados por otros con el fin de mantener el anonimato.

Adicionalmente, se consideró que el material de enseñanza que representó el fenómeno del equilibrio químico, y que el profesor implementó durante el desarrollo de la lección en el grado once, estuviera estructurado fundamentalmente por un conjunto de actividades de aprendizaje de naturaleza experimental y teórica. Así mismo, se determinó utilizar la técnica de animación del Stop Motion a lo largo de las actividades de aprendizaje, como consecuencia a que esta tiene un conjunto de principios de diseño, los cuales les permiten a los estudiantes de forma fácil, diseñar y desarrollar las animaciones a fin de explicar a nivel submicroscópico las entidades y procesos que subyacen al fenómeno del equilibrio químico (Hoban, et al., 2011).

Por otra parte, el análisis de los datos provenientes de las fuentes documentales se focalizó en identificar e interpretar palabras, proposiciones, e imágenes que representan las acciones del profesor y los estudiantes, y los artefactos digitales diseñados por los últimos a lo largo de la lección del equilibrio químico. Para ello, se fundamentó dicha tarea analítica en dos ciclos sucesivos de codificación (Miles, Huberman y Saldaña, 2013). El primer ciclo se caracterizó por comparar de manera constante e iterativa las propiedades de todas las unidades de registro provenientes de las diferentes fuentes documentales, con los principales constructos del marco teórico y la pregunta de investigación (codificación abierta), generando con ello una serie de códigos o temas de carácter inductivo (Véase tabla 1). El segundo ciclo, se apoyó en la clasificación y visualización previa de las unidades de análisis codificadas, con miras a 
reagruparlas en códigos de mayor orden de generalidad, los cuales dieron origen al rango de categorías analíticas que caracterizaron este estudio (Véase tabla 1). Desde luego, este ciclo analítico también permitió realizar una codificación selectiva, la cual brindó la posibilidad de adscribir a las diferentes categorías las unidades de análisis, cuyas propiedades se encontraban alineadas con las características de las categorías inductivas. Esta perspectiva analítica brindó la posibilidad de producir cuatro categorías inductivas, las cuales presentan unas propiedades derivadas de la integración sinérgica entre los diferentes códigos que las configuran (Véase tabla 1).

Tabla 1. Relación entre las categorías y códigos de naturaleza inductiva

\begin{tabular}{|l|l|}
\hline \multicolumn{1}{|c|}{ Categorías } & \multicolumn{1}{c|}{ Códigos de análisis } \\
\hline $\begin{array}{l}\text { El diseño de animaciones en conjunción } \\
\text { con una orientación de enseñanza } \\
\text { sociocultural y fenomenológica, factor } \\
\text { clave en la diferenciación e integración } \\
\text { de los niveles de representación de la } \\
\text { química }\end{array}$ & $\begin{array}{l}\text { Diferenciación integración de los niveles de representación de la } \\
\text { química; dificultades en el aprendizaje del equilibrio químico; } \\
\text { enseñanza de las herramientas conceptuales y prácticas culturales } \\
\text { de la química. }\end{array}$ \\
\hline $\begin{array}{l}\text { El par evaluador de las animaciones } \\
\text { elemento clave en la construcción de la } \\
\text { compresión del equilibrio químico }\end{array}$ & $\begin{array}{l}\text { Estudiantes como evaluadores de las animaciones; evaluación de las } \\
\text { animaciones factor determinante en la comprensión del equilibrio } \\
\text { químico; evaluación focalizada en el contenido y los rasgos } \\
\text { semióticos; identificaciones de los "errores" en el diseño y } \\
\text { desarrollo elemento clave en la comprensión. }\end{array}$ \\
\hline $\begin{array}{l}\text { Las animaciones } \\
\text { cognoscitiva que apoya la visualización, } \\
\text { interpretación y razonamiento de los } \\
\text { procesos químicos }\end{array}$ & $\begin{array}{l}\text { El diseño y desarrollo de animaciones herramientas cognitivas que } \\
\text { distribuyen las demandas de las tareas de visualización; el diseño de } \\
\text { animaciones en el marco de las prácticas experimentales andamia el } \\
\text { proceso cognitivo de la modelización; visualización mediada por las } \\
\text { animaciones digitales; las animaciones ayudan a traducir las } \\
\text { representaciones macroscópicos en submicroscópicas y simbólicas; } \\
\text { y las animaciones herramientas que permiten materializar los } \\
\text { modelos mentales en múltiples representaciones externas. }\end{array}$ \\
\hline $\begin{array}{l}\text { El diseño de las animaciones factor } \\
\text { importante en el desarrollo de las } \\
\text { habilidades densamiento que } \\
\text { demanda la sociedad del conocimiento }\end{array}$ & $\begin{array}{l}\text { Búsqueda, selección, evaluación e interpretación de la información } \\
\text { con el fin de comunicarla; transacción de significados y formas de } \\
\text { significar; y diseño de texto multimodales. }\end{array}$ \\
\hline
\end{tabular}

Fuente: Elaboración propia.

\section{ANÁLISIS Y DISCUSIÓN DE RESULTADOS}

El ciclo sucesivo de codificación realizado al corpus de datos proveniente de las fuentes documentales, permitió inducir un conjunto de categorías cuya discusión teórica apoyada y validada a partir de la evidencia empírica permitió construir una comprensión del caso estudiado. Por todo esto, se puede afirmar que la narrativa que configura a cada una de las siguientes categorías da respuesta a la pregunta de investigación que direcciona este estudio. 


\subsection{El diseño de animaciones en conjunción con una orientación de enseñanza sociocultural y fenomenológica, factor clave en la diferenciación e integración de los niveles de representación de la química}

La literatura en educación en química ha reportado que los estudiantes de la escuela secundaria superior llegan al aprendizaje del equilibrio químico con serias dificultades y concepciones alternativas, como consecuencia a que ellos no son conscientes de la existencia de los tres niveles de representación de la química (macroscópico, submicroscópico y simbólico). Así pues, esta falta de conciencia en el lenguaje de la química obstaculiza la diferenciación e integración de estos tres niveles a lo largo del proceso de modelización de los fenómenos químicos a nivel molecular y simbólico, el cual permite visualizar la naturaleza interactiva y dinámica de las transformaciones químicas reversibles (Candela, 2018; Candela, 2020). Esta restricción se ve reforzada por la acción pedagógica del profesor de química a lo largo del desarrollo de una lección, al moverse de forma inconsciente de un nivel de representación a otro, sobrecargando con este hecho la memoria de trabajo de los aprendices y generándole una ruptura entre el conocimiento nuevo y el que yace en su memoria permanente.

En este sentido, el conjunto de actividades de aprendizaje de naturaleza fenomenológica y modelización molecular que representan el conjunto de ideas, las cuales configuran el fenómeno del equilibrio químico, en conjunción con los razonamientos y acciones pedagógicas del profesor, les permite a los estudiantes diferenciar e integrar los tres niveles de representación de la química (macroscópico, submicroscópico y simbólico). De hecho, al enfrentarlos al inicio de la lección con situaciones fenomenológicas que encarnan las ideas de los factores que afectan la velocidad de una reacción química, junto con las transformaciones reversibles, les permite observar explícitamente las conexiones existentes entre el fenómeno macroscópico, la representación simbólica y su interpretación molecular dinámica. Además, les genera la necesidad de transformar su comprensión expresada inicialmente en términos macroscópico en los otros dos niveles de representación, por medio de diferentes modos semióticos a fin de comunicar un patrón conceptual que le dé solución a la tarea problema previamente formulada (Chang, et al., 2010). Estos presupuestos están coherencia con la siguiente base empírica ubicada en este enlace: https://drive.google.com/file/d/1pxAHFO1xBRzJrJoZlOh-KDw6cDvfNOE8/view?usp=sharing

Así pues, los estudiantes durante el desarrollo de la lección del equilibrio químico abordan las tareas problemas alineadas con el fenómeno macroscópico, por medio de discusiones dentro de un contexto de negociación sociocultural, las cuales se caracterizan por la argumentación sustentada en la evidencia. Naturalmente, el uso del lenguaje oral y escrito en esta clase de actividades de aprendizaje, media el inicio de la externalización y explicitación de los modelos mentales de los aprendices acerca de las ideas que estructuran el fenómeno del equilibrio químico (Schank y Kozma, 2002; Ramírez y Ávila, 2020). También, ellos a fin de representar su interpretación del fenómeno estudiado a nivel molecular hacen uso de la técnica de animación del Stop Motion, que les brinda la posibilidad de integrar múltiples modos semióticos (ej., texto, audio, video, gráfico y animación), con miras a representar las entidades y procesos que subyacen al equilibrio químico en términos submicroscópicos y simbólicos.). Estos presupuestos están coherencia con la siguiente base empírica ubicada en 
De ahí que, se argumente que la técnica de animación del Stop Motion dentro de un contexto experimental y de negociación de significados y formas de significar, estimula a los estudiantes a identificar los enlaces de referencia entre los tres niveles de representación de la química (Hoban, et al., 2011). Desde luego, para dar sentido a estas representaciones, ellos deben comprometerse activamente en discusiones reflexivas sobre las observaciones e inferencias provenientes de la interacción con el fenómeno, y establecer relaciones semánticas entre los niveles de representación macroscópico, submicroscópico y simbólico que subyacen al fenómeno del equilibrio químico. Naturalmente, estas acciones inteligentes son complementadas y asistidas por las potencialidades que ofrece la técnica de animación multimedia del Stop Motion, las cuales les permite construir un modelo dinámico de los procesos moleculares que fundamentan, por ejemplo, las transformaciones químicas de carácter reversibles (Kozma, 2000). En la siguiente viñeta se presenta una secuencia de algunos fotogramas extraídos de una de las animaciones diseñadas por el estudiante Diego (Véase figura 1):

1.
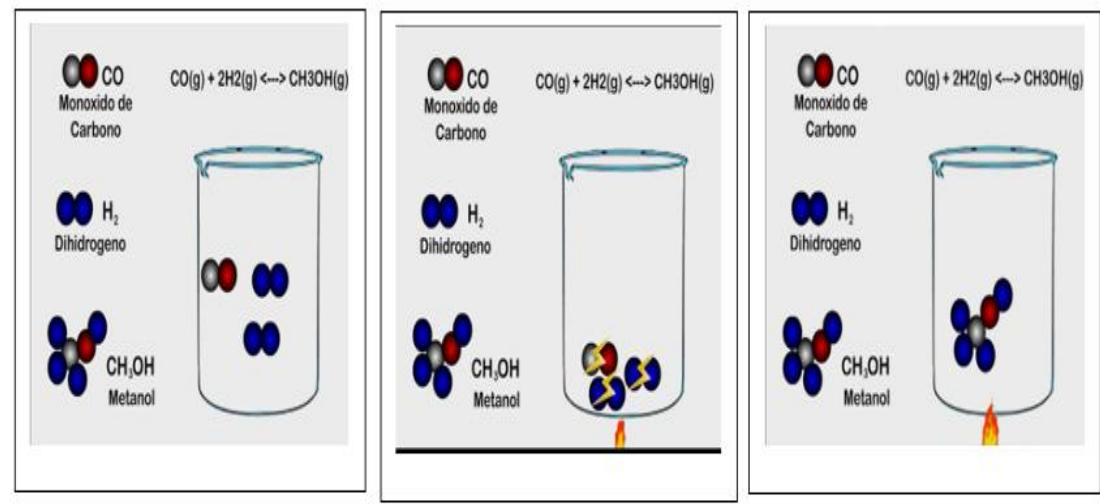

4.

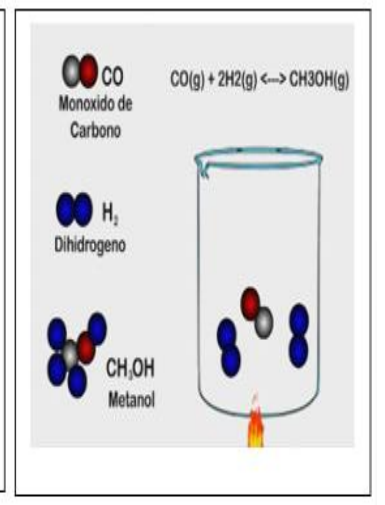

Figura 1. Fotogramas realizados por un estudiante.

\subsection{El par evaluador de las animaciones elemento clave en la construcción de la compresión del equilibrio químico}

El par evaluador (estudiantes) dentro de un contexto de negociación de significados ha comenzado a ser considerado, uno de los factores que andamia a los estudiantes durante la construcción de la comprensión del equilibrio químico. Desde luego, esta tarea analítica les brinda la oportunidad de establecer relaciones semánticas y sintácticas entre el fenómeno macroscópico, los modelos moleculares dinámicos y las representaciones simbólicas, con la intención de explicar y predecir el fenómeno químico bajo consideración. Para ello, el par evaluador-estudiante se focaliza en determinar las fortalezas y debilidades de dichas relaciones, tanto a nivel del contenido o referente fenomenológico representado, como en los modos de representación moleculares y simbólicos que permiten visualizar las transformaciones químicas reversibles. 
En el mismo sentido, la evaluación por pares ayuda a los estudiantes a analizar una serie de criterios que indican la buena calidad del artefacto involucrado en la tarea de aprendizaje, y de esta forma utilizar las sugerencias emergidas desde este contexto de negociación de significados y formas de significar en sus propias animaciones fenomenológicas. Adicionalmente, este proceso analítico les permite internalizar la idea que todo texto de carácter científico ya sea de naturaleza impresa o digital, debe ser sometido a un par evaluador con la intención de acercar esta representación semiótica a los modelos científico consensuados (Chang, et al., 2010). Estas asunciones están en coherencia con la viñeta ubicada en el siguiente enlace: https://drive.google.com/file/d/1KEDrhMlx84T7mFtPBUucoQHTPPD3EOZE/view?usp=sharing

Finalmente, como lo argumentan Chang, et al. (2010) las actividades de aprendizaje donde los estudiantes diseñan, desarrollan y evalúan de manera reflexiva las animaciones, resultan ser estrategias pedagógicas efectivas a lo largo de la construcción de una comprensión del fenómeno del equilibrio químico. De hecho, en este caso los aprendices quienes someten a evaluación colegiada sus animaciones de procesos moleculares, logran identificar y volver conscientes las concepciones alternativas representadas en la configuración semiótica de la animación. Esta situación, les permite alcanzar una interpretación significativa, la cual apoya el rediseño de la animación a través del uso de las múltiples representaciones, y el establecimiento de conexiones entre los tres niveles de representación que subyacen al equilibrio químico (macroscópico, submicroscópico y simbólico).

\subsection{Las animaciones herramienta cognoscitiva que apoya la visualización, interpretación y razonamiento de los procesos químicos}

Los razonamientos y acciones del profesor y los estudiantes a lo largo de la implementación de la lección del equilibrio químico, permiten evidenciar que la tecnología digital no debe apoyar el aprendizaje intentando instruir al estudiante, sino, más bien dichos recursos digitales tienen que ser utilizados como herramientas cognoscitivas que median la construcción del conocimiento como un capital individual y social. De ahí que, se haya comenzado a considerar el diseño y desarrollo de animaciones a través de la técnica de animación del Stop Motion, como herramientas cognoscitivas de naturaleza abstracta y concreta, las cuales en conjunción con las acciones del profesor andamian a los estudiantes en la construcción de una comprensión de cada una de las ideas que configuran el fenómeno del equilibrio químico. En este sentido, estas herramientas funcionan como socias del pensamiento, extendiendo las capacidades cognoscitivas de los estudiantes más allá de los simples medios de entrega de información (Salomon, Perkins y Globerson, 1991).

Así mismo, el diseño de las animaciones del equilibrio químico a través de la técnica del Stop Motion apoyada en el software de Fireworks, les permite continuar a los estudiantes desarrollando las habilidades de la modelización de los fenómenos químicos a nivel molecular (Chang y Quintana, 2006). Conviene subrayar, que estas habilidades son reconocidas como un elemento clave de la experticia en el campo de la química y la educación en química, considerando que, en el primero ayuda en la construcción de los modelos teóricos de esta disciplina, en cuanto en el segundo, media el aprendizaje de los diferentes contenidos. Así pues, la modelización apoyada en la experimentación y en la técnica de visualización del Stop Motion dentro de un contexto sociocultural, asiste a los estudiantes durante la construcción 
de representaciones dinámicas y moleculares del mundo submicroscópico y simbólico que encarna cada una de las ideas del equilibrio químico, con miras a externalizar sus modelos mentales del fenómeno bajo consideración. Este presupuesto se encuentra en coherencia con la siguiente viñeta ubicada en el enlace:https://drive.google.com/file/d/1SJfOXGEYy1353oLHowN3j12XsLiVRYN/view? usp=sharing

La estrategia del diseño y desarrollo de animaciones dentro de un contexto de transacción de significados y experimentación, sirve como una herramienta de pensamiento y aprendizaje de la química. Desde luego, esta ayuda a los estudiantes a desarrollar las capacidades de visualización, las cuales ejercen una fuerte influencia en la traducción de los modelos mentales originados desde la experimentación en múltiples representaciones externas (p. ej., animaciones) que representan los modelos teóricos de la química escolar. En este sentido, los estudiantes actúan como los diseñadores de las múltiples representaciones externas, que encarnan a nivel submicroscópico las transformaciones químicas de las sustancias, y la técnica del Stop Motion, como una "herramienta cognoscitiva" que ayuda a organizar y representar el conocimiento con miras a que otros puedan comprenderlo. Evidencia de las anteriores asunciones se pueden ver en la modelización del equilibrio químico del $\mathrm{NH}_{3}$ realizada por un grupo de estudiantes del grado 11-2 (véase el link https://youtu.be/Y7jfJSqZRJA?t=20).

Por otra parte, los estudiantes durante el diseño de las animaciones mantienen la atención en el comportamiento dinámico de la reacción química reversible, asociada al fenómeno experimental puesto en escena en el aula. Probablemente, esta situación les permite razonar sobre las entidades moleculares y procesos que subyacen a las reacciones químicas en equilibrio dinámico (Schank y Kozma, 2002). De donde resulta que la tarea de diseñar animaciones que encarnan a nivel submicroscópico y simbólico el conjunto de observaciones obtenidas desde la experimentación, facilita la interpretación y el razonamiento de algunas de las ideas que estructuran el fenómeno químico. De hecho, esta clase de tarea cognitiva de la modelización, les brinda a los estudiantes la oportunidad de establecer discusiones reflexivas sobre las múltiples representaciones externas usadas, con miras a representar los modelos mentales y los aspectos técnicos de traducción de éstos en un artefacto digital. Este presupuesto se encuentra en coherencia con la siguiente viñeta ubicada en el enlace: https://drive.google.com/file/d/1GwrTwuZZ7H9s4Uau5xfSKGOlxiYYP 2p/view? usp=sharing

\subsection{El diseño de las animaciones factor importante en el desarrollo de las habilidades de pensamiento que demanda la sociedad del conocimiento}

La tarea del diseño y desarrollo de las animaciones que representan las diferentes ideas del fenómeno equilibrio químico, demanda de los estudiantes aspectos cognitivos como: observación consciente de los principales aspectos que caracterizan la situación fenomenológica; discusión colegiada de los problemas que subyacen al fenómeno; búsqueda, selección, y evaluación de información alineada al equilibrio químico; y construcción de un artefacto cognitivo con miras a comunicar y diseminar la comprensión alcanzada (Candela, 2018). Naturalmente, estas acciones inteligentes requieren de los estudiantes el haber comenzado a desarrollar las habilidades que demanda la actual sociedad del conocimiento, las cuales les permiten gestionar de manera autónoma la construcción de un determinado conocimiento a fin de dar solución a problemáticas particulares. 
Por todo esto, se considera que el vínculo de la experimentación que representa el equilibrio químico en conjunción con el diseño de animaciones desde una perspectiva cognitiva, y la orientación sociocultural del profesor, asisten a los estudiantes de grado once en el desarrollo de habilidades, tales como: aprender a aprender a lo largo de la vida; búsqueda, selección e interpretación de información; razonamiento lógico-matemático; comunicación a través del lenguaje multimedia, y relaciones interpersonales. Desde luego, estas habilidades les permiten utilizar la experimentación, la internet y el software del Fireworks que apoya a la técnica del Stop Motion, con el propósito de construir una comprensión de cada una de las ideas que configuran el equilibrio químico. Para ello, ubican y evalúan críticamente la utilidad de la información, y posteriormente la interpreta con miras a dar solución a las tareas problemas y, a representar la comprensión fenomenológica alcanzada por medio de textos multimediales, los cuales son sometidos a la evaluación por parte de sus compañeros de clase. Por consiguiente, el desarrollo de dichas habilidades de pensamiento resulta ser necesarias para que los estudiantes aprendan, trabajen, interactúen socialmente y hagan frente a las necesidades personales y profesionales en la actual sociedad del conocimiento. Estas asunciones son recogidas en un fragmento de la entrevista realizada a Diego acerca de la estrategia de diseño de la animación. Este presupuesto se encuentra en coherencia con la siguiente viñeta ubicada en enlace: https://drive.google.com/file/d/1B1hDc 9hpaw5YgyN4Cs71h INARRWYxg/view?usp=sharing

\section{CONCLUSIONES}

Esta investigación determina que el recurso curricular del diseño de animaciones, puede ser utilizado dentro de un contexto sociocultural y experimental, como una herramienta de pensamiento y aprendizaje del equilibrio químico, y no simplemente como un medio para presentar contenidos de forma unidireccional a los estudiantes de grado once (Chang y Quintana, 2006; Chang, et al., 2010). Desde luego, las potencialidades brindadas por la técnica de animación del Stop Motion, ayudan a distribuir las demandas cognitivas entre los estudiantes y el recurso digital, las cuales subyacen a los procesos de modelización de las reacciones químicas reversibles y el principio de Le Chatelier. Esta situación les permite a los estudiantes continuar extendiendo los procesos de visualización, interpretación y razonamiento que caracterizan a la educación en química, junto con el desarrollo de una alfabetización informática requerida por la actual sociedad del conocimiento.

El desarrollo de la lección deja ver claramente que la estrategia del par evaluador-estudiante apoya la construcción progresiva de la comprensión de las ideas que configuran el equilibrio químico. Así pues, los aprendices a lo largo del desarrollo de la lección someten a evaluación formativa, las animaciones o visualizaciones digitales diseñadas y desarrolladas, como consecuencia a los procesos de negociación de significados y formas de significar en el marco de la experimentación. De hecho, la acción de evaluar las animaciones en términos de los procesos moleculares que fundan al fenómeno y los modos semióticos que los representan, asisten a los estudiantes en el establecimiento de relaciones de significado entre las observaciones sensoriales y las múltiples representaciones externas a nivel submicroscópico, a fin de comunicar un patrón conceptual a sus compañeros (Chang, et al., 2010). Por otro lado, se determina que la implementación de la perspectiva del par evaluador-estudiante demanda una gran cantidad de tiempo, sin embargo, también se evidencia que dicha 
inversión es significativa para el aprendizaje comprensivo de un fenómeno complejo como lo es el equilibrio químico.

\section{REFERENCIAS}

Candela, B. F. (2018). El lenguaje y las múltiples representaciones externas. Estrategias de pensamiento en el aprendizaje de las ciencias. Programa Editorial Universidad del Valle.

Candela, B. F. (2020). Oralidad, lectura y escritura competencias mediadoras del aprendizaje del currículo de Química: el caso del equilibrio químico. Revista Científica, 1(37), 1829. DOI: https://doi.org/10.14483/23448350.14839

Candela, B. F., y Viáfara, R. (2014). Aprendiendo a enseñar química. Programa Editorial Universidad del Valle.

Chan, M. S., y Black, J. B. (2005). When can animation improve learning? Some implications for human computer interaction and learning. Proceedings of the World Conference on Educational Multimedia, Hypermedia and Telecommunications 2005 (pp. 2581-2588). Norfolk, VA.

Chang, H.-Y. y Quintana, C. (2006). Student-generated animations: Supporting middle school students' visualization, interpretation and reasoning of chemical phenomena. Proceedings of the 7th International Conference of the Learning Sciences (pp. 71-177). International Society of the Learning Sciences, Bloomington, IN.

Chang, H.-Y., Quintana, C., y Krajcik, J. S. (2010). The impact of designing and evaluating molecular animations on how well middle school students understand the particulate nature of matter. Science Education, 94 (1), 73-94. https://doi.org/10.1002/sce.20352

Chiu MH., y Wu HK. (2009) The Roles of Multimedia in the Teaching and Learning of the Triplet Relationship in Chemistry. En: Gilbert J.K., Treagust D. (eds) Multiple Representations in Chemical Education. Models and Modeling in Science Education, vol 4. Springer, Dordrecht. https://doi.org/10.1007/978-1-4020-8872-8_12

Hoban, G. y Nielsen, W. (2010). The 5 Rs: A new teaching approach to encourage slowmations (student-generated animations) of science concepts. Teaching Science: Australian Science Teachers Journal, 56 (3), 33-37. https://ro.uow.edu.au/edupapers/178

Hoban, G., Loughran, J. y Nielsen, W. (2011). Slowmation: preservice elementary teachers representing science knowledge through creating multimodal digital animations. Journal of Research in Science Teaching, 48(9), 985-1009. https://doi.org/10.1002/tea.20436

Hubscher-Younger, T., y Narayanan, N. H. (2003). Dancing hamsters and marble statues: characterizing student visualizations of algorithms. En proceedings ACM symposium on Software visualization. San Diego, CA. https://doi.org/10.1145/774833.774847 
Kozma, R. (2000). Representation and language: The case for representational competence in the chemistry curriculum. En proceedings 16th Biennial Conference on Chemical Education. https://doi.org/10.1021/ed077p1390

Miles, M. B., Huberman, A. M. y Saldaña, J. (2013). Qualitative data analysis: a methods sourcebook. SAGE.

Ramírez, M. H. y Ávila, G. (2020). La enseñanza de la energía cinética a través de juguetes tradicionales y la modelización en el bachillerato en México. Edutec. Revista electronica de Tecnología Educativa, 72, 88-111. https://doi.org/10.21556/edutec.2020.71.1559

Salomon, G., Perkins, D.N. y Globerson, T. (1991). Partners in cognition: extending human intelligence with intelligent technologies. Educational researcher, 20(3). 2-9. https://doi.org/10.3102/0013189X020003002

Schank, P. y Kozma, R. (2002). Learning Chemistry Through the Use of a Representation-Based Knowledge Building Environment. Journal of Computers in Mathematics and Science Teaching, 21(3), 253-279.

Vermaat, H., Kramers-Pals, H., y Schank, P. (2003). The use of animations in chemical education. En Proceedings of the International Convention of the Association for Educational Communications and Technology (pp. 430-441). Anaheim, CA.

Weiss, R. E., Knowlton, D. S., y Morrison, G. R. (2002). Principles for using animation in computer-based instruction: theoretical heuristics for effective design. Computers in Human Behavior, 18 (4) 465-477. https://doi.org/10.1016/S0747-5632(01)00049-8

Yin, R.K. (2003). Investigación sobre estudio de casos. Diseño y Métodos. Sage.

\section{Para citar este artículo:}

Candela, B. F. (2021). El diseño y desarrollo de animaciones como estrategia que ayuda a mediar la comprensión del equilibrio químico en la escuela. Edutec. Revista Electrónica De Tecnología Educativa, (75), 124-136. https://doi.org/10.21556/edutec.2021.75.1787 